\title{
Ultraviolet Absorption Spectra of Seven Substituted Benzenes
}

\author{
By Ralph Stair
}

\begin{abstract}
The ultraviolet absorption spectra of seven substituted benzenes have been measured in the region of 220 to 300 millimicrons. The substances were $n$-, iso-, sec-, and tert-butylbenzene and 1,2-, 1,3-, and 1,4-diethylbenzene. The wavelengths (also frequencies in centimeters $^{-1}$ ) are shown for the absorption bands for each substance. Spectral absorbancy curves are given for the same concentration in each case. The hydrocarbons were NBS Standard Samples prepared in connection with American Petroleum Research Project 6 and were highly purified in the Chemistry Division of this Bureau. These spectral data should be of value in chemical analysis in the field of ultraviolet absorption spectroscopy.

The measurements were made with a Beckman quartz spectrophotometer on 1-centimeter cells of the hydrocarbons in a solution of isooctane (2,2,4-trimethylpentane).
\end{abstract}

\section{Introduction}

This work is an extension of the contribution [1] ${ }^{1}$ of the radiometry laboratory of this Bureau in the cooperative research program with the American Petroleum Institute Research Project 44 in the study of the absorption spectra of the constituents of petroleum that have been extensively purified. This report deals with the liquid phase of two series of compounds, butylbenzenes and diethylbenzenes, that are closely related and that have significant selective absorption in the ultraviolet region between 220 and $280 \mathrm{~m} \mu$.

No attempt has been made, in this report, to give a detailed theoretical interpretation of the structural features of the observed spectra. Primary emphasis has been placed on the precise mapping of these features in order to make available reference data [5] for analytical purposes. Particular attention is called to the similarities between these spectra of closely related compounds and to the small displacements of analogous features associated with the substitution of different chemical groups in the benzene structure.

There is within the published literature a vast amount of empirical data on the spectra of com-

${ }_{1}$ Figures in brackets indicate the literature references at the end of this paper. plex molecules - especially in the liquid phaseand it has been fairly well established that the general appearance and intensity of an ultraviolet absorption spectrum depend upon the nature of the electronic states between which the electronic transition takes place. Each transition is presumed to be associated with a characteristic vibrational structure of the molecule, which is fundamentally different for "allowed" and "forbidden" transitions [6, 7]. Nevertheless, the interpretation of the spectra is often quite difficult because of lack of complete knowledge of the vibrations of the molecule in the ground state, and because in many cases bands can be interpreted in different ways, resulting in an ambiguity that makes such an interpretation meaningless [8] and confusing to other workers in the field.

Probably more is known about the structure of benzene and some of the simple benzene derivatives than any other polyatomic molecules. In any case, benzene appears to be unique in a number of characteristics and has undergone many investigations in the liquid, vapor, and solid phases by investigators in the ultraviolet and infrared, as well as for Raman and fluorescence spectra for both the regular and the deuterosubstituted material. 


\section{Instrument and Method of Measure- ment}

A Beckman quartz-spectrophotometer [2, 3] was employed, without modification, except that it had been specially supplied with a "blue sensitive" phototube in an envelope of Corning 9740 glass. By using a hydrogen lamp in an envelope of similar glass, measurements were possible down to 200 $\mathrm{m} \mu$.

In this spectrophotometer the photometric ("density," equivalent to absorbancy) scale is based on electrical principles, in that the photoelectric current through a load resistor produces a voltage drop that is balanced by a potentiometer, thus giving a null reading for each condition of balance. To make this null setting possible, any voltage unbalance is electronically amplified and impressed across the terminals of a sensitive direct-current milliammeter. The precision of the instrument thus becomes largely a function of the linearity of the potentiometer circuits and the irradiation-current characteristic of the vacuum caesium-antimony silver phototube employed. Since the operation of the instrument is based on these principles and conditions, a high degree of precision and accuracy may be expected when care is taken in its use.

In this work the hydrocarbons were examined in the liquid phase. Five concentrations of each material were employed, and the absorbancy observed at intervals of 0.5 to $1.0 \mathrm{~m} \mu$ throughout the range from 220 to about $280 \mathrm{~m} \mu$ for all values of absorbancy less than about 1.0.

The concentration of the hydrocarbon was determined by carefully weighing about $0.5 \mathrm{~g}$ of the pure material and then diluting it in isooctane (2,2,4-trimethylpentane). For example, to obtain the $10 \mathrm{~g}$ /liter solution the procedure was as follows: First a 50 ml-flask containing a few cubic centimeters of isooctane was placed in the balance and carefully weighed. Then a $0.5-\mathrm{g}$ weight was placed on the opposite pan and the hydrocarbon. carefully added (in small drops) until the amount slightly exceeded the required $0.5 \mathrm{~g}$. Next, a careful balance was obtained to determine the exact amount of hydrocarbons added-for example, $0.503 \mathrm{~g}$. Finally, the flask was filled with isooctane to the $50 \mathrm{ml}$ mark, after which an additional $0.3 \mathrm{ml}$ was added to give the total of $50.3 \mathrm{ml}$ to produce a $10 \mathrm{~g} /$ liter solution. The other concentra- tions were simply obtained by diluting samples of the $10 \mathrm{~g} /$ liter solution. All operations were performed at room temperature, with the flasks kept "stopped" to prevent errors due to selective evaporation. The prepared samples were kept in a refrigerator until immediately preceding the filling of the absorption cells.

The reflection loss from the absorption-cell windows and the possible absorption by the isooctane solution were annulled by using a comparison cell filled only with isooctane taken from the same container as that employed in preparing the hydrocarbon solutions. Thus the data recorded here are the specific absorbancies of the concentrations of the hydrocarbons employed.

All of the substances that were measured were obtained from F. D. Rossini of the Chemistry Division of this Bureau. They were purified under his direction in connection with the American Petroleum Institute Research Project No. 6. The substances studied and the amount of impurity in each one is given in table 1 [4]. The absorptionspectra data on the butylbenzenes and diethylbenzenes have been made a part of the catalog of ultraviolet spectrograms of the American Petroleum Institute Research Project No. 44 [16].

Table 1. Purity of compounds

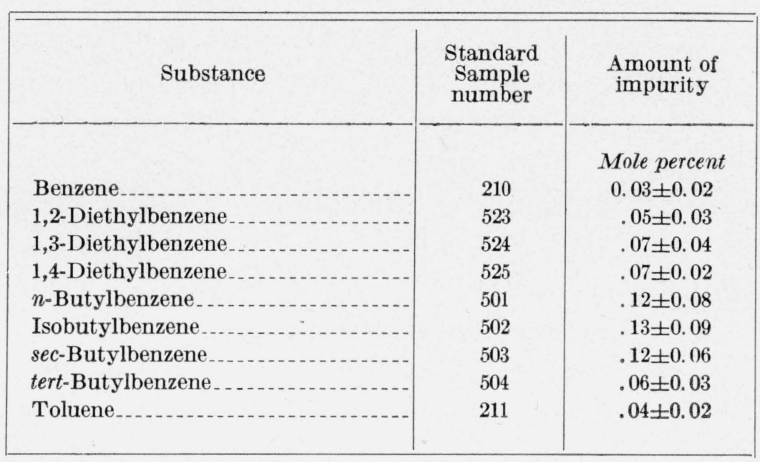

\section{Results and Discussion}

The ultraviolet spectral absorbancies of each of the seven substituted benzenes were measured at room temperature in solutions having concentrations of $10.0,1.0,0.5,0.2$, and $0.1 \mathrm{~g} /$ liter (all for a 1-cm cell length). In all cases the results for the different concentrations were in close agreement with Beer's law, indicating that for these hydrocarbons no change in chemical or physical constitution occurs as the result of association or 
interaction upon placing them in an isooctane solution. It was therefore felt unnecessary to display in the illustrations and tables data for more than one concentration (that for $0.5 \mathrm{~g} /$ liter), since any other values can be calculated from the recorded data in accordance with Beer's law. The spectral absorbancy data for a concentration of 0.5 $\mathrm{g} /$ liter were chosen for purposes of illustration because, in general, they were within the absorption range (specific absorbancy 0.2 to 0.8 ) most suitable for analysis considerations (see figs. 1 and 2 and tables 2 and 3 ).

The analysis of the ultraviolet absorption spectrum of benzene [10] has shown the vibrational structure to be in agreement with the selection rules for the forbidden electronic transition $A_{1 g} \rightarrow$ $B_{2 u}$. It is held that this transition is rendered possible through the distortion of the benzene molecule by $E_{o}^{+}$vibrations. Each of the eight absorption bands (see fig. 1) results from a series of more or less superimposed lines or bands. Sponer, et. al [10] list eight numerical equations principally in terms of the 0,0 band frequency $\left(38,089 \mathrm{~cm}^{-1}\right)$, and the totally symmetrical carbon vibrations of $992 \mathrm{~cm}^{-1}$ and $923 \mathrm{~cm}^{-1}$ belonging to the ground and upper states, respectively, of the benzene molecule, which define the positions of the integral parts of these absorption bands (in the vapor state). The uniform spacing of $923 \mathrm{~cm}^{-1}$ of the principal component (the $A$ series) of these bands is indicative of the important part played by the totally symmetrical vibrations of the benzene molecule in the excited state.

When a substitution has been made for one or more of the $\mathrm{H}$ atoms of the benzene ring, the symmetry is changed from the type $D_{6 n}$ to another, depending upon the particular substituent. Often the substituent, as is the case in the present work, contains groups such as $\mathrm{CH}, \mathrm{CH}_{2}, \mathrm{CH}_{3}$, which have mobile electrons that can resonate or migrate into the benzene ring and thus produce a continuum or other special characteristic in the absorption spectrum.

All of the substituted benzenes herein reported



Figure 1. Spectral absorbancies of benzene, toluene, and the four butylbenzenes, all for a concentration of $0.5 \mathrm{~g} /$ liter, cell thickness $1.000 \mathrm{~cm}$.

Although the ordinates are displaced for the different curves, all are reduced to the same scale. 


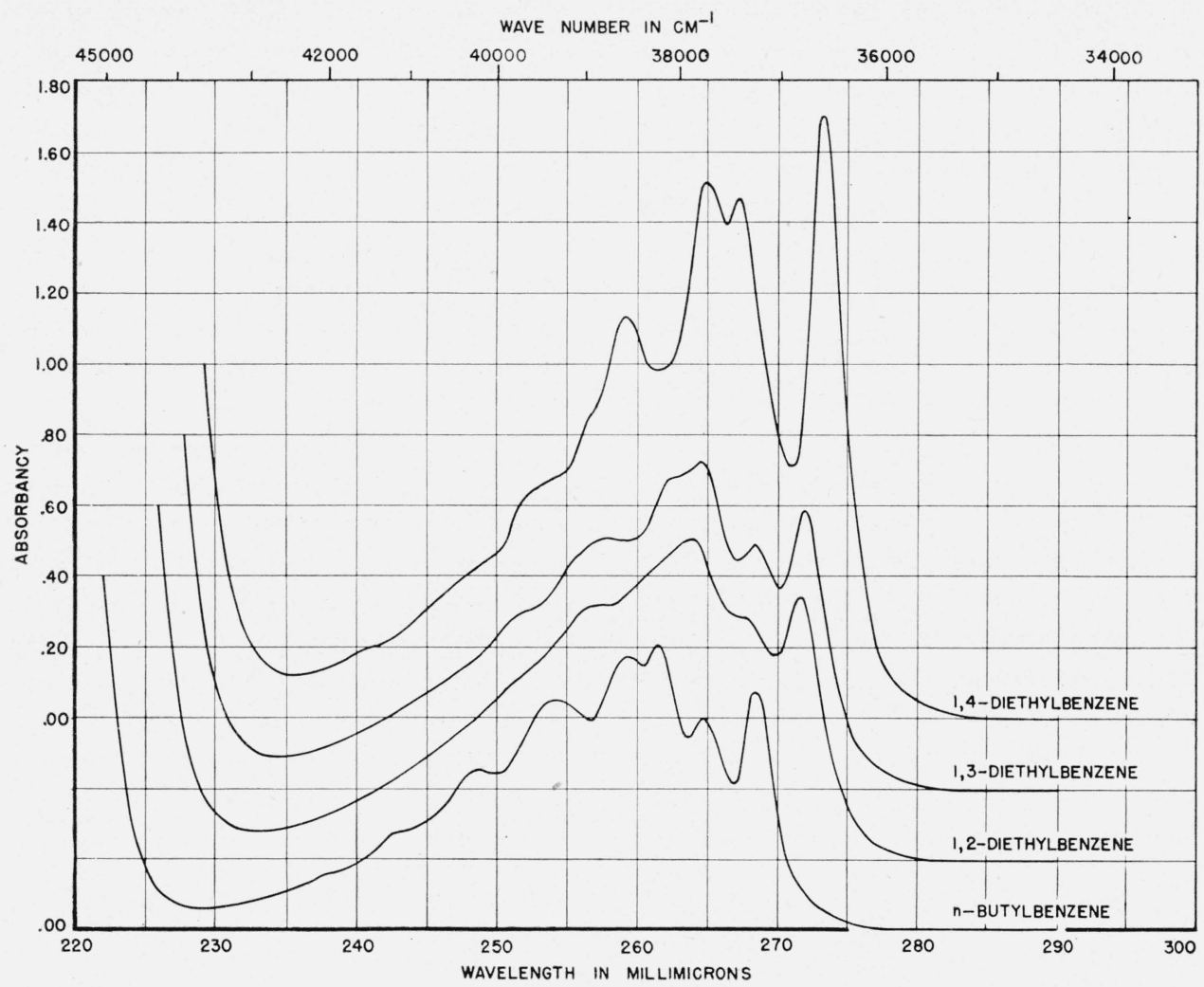

Figure 2. Spectral absorbancies of n-butylbenzene and the three diethylbenzenes, all for a concentration of $0.5 \mathrm{~g} / \mathrm{liter}$, cell thickness $1.000 \mathrm{~cm}$.

Although the ordinates are displaced for the different curves, all are reduced to the same scale.

have similar absorption characteristics in the ultraviolet. In all cases the spectra are shifted nearer to the visible than that for benzene. Also it appears that all the materials have a double set of spectra $[12,13]$. One of these corresponds to the $A$ series in benzene. The other (denoted as $M$ series below) is probably the result of electron migration or other change in the symmetry of the molecule, since it does not occur within a frequency range to be associated with any of the eight series for benzene. Since a major portion of the spectra of all of these substituted benzenes is carried over from benzene, it appears that the mechanism that brought the benzene spectrum into existence remains relatively important. Furthermore, since the carbon atoms enter into the important vibrations responsible for the benzene spectrum the benzene ring must undergo only slight perturbations for the butyl- and diethyl-substitutions. The general shift of the spectra toward longer wavelengths (lower frequencies) is indicative of slight spatial increases between the carbon atoms in the ring structure and to the increase in mass of the substituted molecule.

The spectrum of liquid $n$-butylbenzene at room temperature (about $24^{\circ} \mathrm{C}$ ) is shown in both figures 1 and 2. It is apparently composed of two series of bands, designated $A$ and $M$ in this report. The $A$ series, presumably corresponding to the $A$ series in benzene, has its first band located at approximately $37,780 \mathrm{~cm}^{-1}$ (see table 3 ) with a second member spaced at an interval of about $770 \mathrm{~cm}^{-1}$ to higher frequencies. The $M$ series has a 0,0 band at a frequency of about $37,240 \mathrm{~cm}^{-1}$ with a second member spaced at an interval of about $990 \mathrm{~cm}^{-1}$ toward the higher frequencies. Only the first two pairs of bands are resolved, since they approach each other toward shorter wavelengths and are no doubt further complicated through overlapping of spectra from weaker series, band splitting, rotational effects, etc. The ratio of the intensities of the $M$ to the $A$ bands appears to decrease with frequency.

For comparative purposes the absorption spec- 
tra of the four butylbenzenes are placed together with those for liquid benzene and toluene in figure 1. The marked similarity between the four butylbenzenes and toluene is impressive. Whereas the spectra are similar, their differences are real and easily measured. In general there is a progressive decrease in absorbancy in the order; $n$-, iso-, sec-, tert-butylbenzene. It is interesting to note the gradual shift in the position of the important absorption bands to higher frequencies in progressing toward the more complicated molecules in this series of monosubstituted benzenes from the simple toluene to tert-butylbenzene. In some respects the absorption spectrum of tert-butylbenzene appears to be more like that of benzene than that of the others of this group.

The spectra of the 1,2-, 1,3-, and 1,4-diethyl-

TABLE 2. Values of spectral ultraviolet absorbancy for a 0.5-g/liter solution of the seven substituted benzenes, cell thickness $1.000 \mathrm{~cm}$

\begin{tabular}{|c|c|c|c|c|c|c|c|}
\hline Wavelength & $n$-Butylbenzene & Isobutylbenzene & sec-Butylbenzene & tert-Butylbenzene & $\begin{array}{l}\text { 1,2-Diethylben- } \\
\text { zene }\end{array}$ & $\begin{array}{l}\text { 1,3-Diethylben- } \\
\text { zene }\end{array}$ & $\begin{array}{l}\text { 1,4-Diethylben- } \\
\text { zene }\end{array}$ \\
\hline \multicolumn{8}{|l|}{$\begin{array}{l}m \mu \\
222\end{array}$} \\
\hline 222 & 0.990 & -.... & 0.717 & 0.582 & & & \\
\hline 224 & .317 & 0.418 & .242 & .198 & - & - & - \\
\hline 226 & .111 & .138 & .087 & .079 & 0.960 & - non & - \\
\hline 228 & .063 & .066 & .052 & .053 & .308 & 0.866 & (n) \\
\hline 230 & .062 & .058 & .056 & .061 & .123 & .296 & 0.642 \\
\hline 232 & .079 & .072 & .070 & .074 & .081 & .126 & .236 \\
\hline 234 & .096 & .089 & .091 & .095 & .087 & .094 & .137 \\
\hline 236 & .128 & .119 & .123 & .128 & .106 & .100 & .127 \\
\hline 238 & .161 & .153 & .149 & .151 & .134 & .124 & .146 \\
\hline 240 & .192 & .182 & 190 & .198 & 169 & .157 & .185 \\
\hline 241 & .220 & .210 & .223 & .228 & .189 & .174 & .202 \\
\hline 242 & .259 & .246 & .253 & .245 & .210 & .195 & .218 \\
\hline 243 & .284 & .272 & .266 & .251 & .237 & .218 & .242 \\
\hline 244 & .291 & .282 & .274 & .272 & .266 & .244 & .275 \\
\hline 245 & .305 & .297 & .302 & .313 & .293 & .274 & .312 \\
\hline 246 & .340 & .327 & .347 & .354 & .321 & .296 & .342 \\
\hline 247 & .396 & .376 & .399 & .386 & .350 & .323 & .383 \\
\hline 248 & .452 & .428 & .424 & .389 & .379 & .350 & .412 \\
\hline 249 & .459 & .438 & .417 & .381 & .416 & .382 & .438 \\
\hline 250 & .452 & .434 & .424 & .413 & .460 & .427 & .472 \\
\hline 251 & .473 & .457 & .483 & .500 & .499 & .470 & .550 \\
\hline 252 & .554 & .527 & .565 & .551 & .532 & .498 & .629 \\
\hline 253 & .628 & .600 & .601 & .545 & .564 & .514 & .658 \\
\hline 254 & .648 & .619 & .598 & .518 & .602 & .558 & .678 \\
\hline 255 & .647 & 602 & .570 & .480 & 652 & .620 & .702 \\
\hline 256 & .620 & .580 & .540 & .497 & .703 & .668 & .790 \\
\hline 257 & .600 & .572 & .600 & .616 & .722 & .694 & .884 \\
\hline 258 & .695 & .650 & .700 & .681 & .720 & .708 & .969 \\
\hline 259 & .768 & .740 & .722 & .641 & .742 & .704 & 1. 09 \\
\hline 260 & .768 & .740 & .700 & .598 & .790 & .708 & 1. 07 \\
\hline 261 & .774 & .706 & .689 & .533 & .826 & .782 & 1.00 \\
\hline 262 & .790 & .693 & .576 & .428 & .856 & .868 & .99 \\
\hline 263 & .610 & .574 & .488 & .431 & .900 & .887 & 1.05 \\
\hline 264 & .559 & .523 & .518 & .495 & .905 & .910 & 1. 32 \\
\hline 265 & .596 & .564 & .508 & .451 & .839 & .899 & 1. 50 \\
\hline 266 & .492 & .496 & .427 & .380 & .734 & .746 & 1. 40 \\
\hline 267 & .420 & .416 & .452 & .378 & .692 & .644 & 1.45 \\
\hline 268 & .611 & .484 & .495 & .330 & .686 & .681 & 1. 33 \\
\hline 269 & .600 & .509 & .352 & .206 & .617 & .650 & 1.04 \\
\hline 270 & .352 & .334 & .187 & .113 & .578 & .568 & .816 \\
\hline 272 & .095 & .098 & .063 & .048 & .706 & .785 & .958 \\
\hline 274 & .043 & .046 & .029 & .021 & .276 & .332 & 1. 420 \\
\hline 276 & .014 & .017 & .010 & .008 & .087 & .102 & .444 \\
\hline 278 & .004 & .007 & .002 & .003 & .033 & .038 & .129 \\
\hline
\end{tabular}


TABLE 3. Frequencies in centimeters ${ }^{-1}$ (also wavelengths in millimicrons) of the first 2 bands of each absorption spectra system

A bsorbancies of a $0.5-\mathrm{g} /$ liter solution, thickness $1.000 \mathrm{~cm}$

\begin{tabular}{|c|c|c|c|c|c|c|c|c|c|c|c|c|}
\hline \multirow{2}{*}{ Substance } & \multicolumn{3}{|c|}{ First M band } & \multicolumn{3}{|c|}{ Second $\mathrm{M}$ band } & \multicolumn{3}{|c|}{ First A band } & \multicolumn{3}{|c|}{ Second A band } \\
\hline & $\mathrm{m}_{\mu}$ & $\mathrm{cm}^{-1}$ & $\begin{array}{l}\text { Absorb- } \\
\text { ancy }\end{array}$ & $\mathrm{m}_{\mu}$ & $\mathrm{cm}^{-1}$ & $\begin{array}{l}\text { Absorb- } \\
\text { ancy }\end{array}$ & $\mathrm{m}_{\mu}$ & $\mathrm{cm}^{-1}$ & $\begin{array}{l}\text { Absorb- } \\
\text { ancy }\end{array}$ & $\mathrm{m}_{\mu}$ & $\mathrm{cm}^{-1}$ & $\begin{array}{l}\text { Absorb- } \\
\text { ancy }\end{array}$ \\
\hline$n$-Butylbenzene & 268.5 & 37240 & 0.677 & 261.6 & 38230 & 0.812 & 264.7 & 37780 & 0.600 & 259.4 & 38550 & 0.780 \\
\hline Isobutylbenzene $\ldots \ldots \ldots \ldots$ & 268.7 & 37220 & .538 & 261.5 & 38240 & .710 & 265.0 & 37740 & .570 & 259.4 & 38550 & .750 \\
\hline sec-Butylbenzene & 267.8 & 37340 & .508 & 260.3 & 38420 & .703 & 264.3 & 37840 & .533 & 258.6 & 38670 & .725 \\
\hline tert-Butylbenzene & 267.2 & 37420 & .381 & a 260.3 & 38420 & .580 & 264.2 & 37850 & .500 & 258.0 & 38760 & .683 \\
\hline 1,2-Diethylbenzene & 271.6 & 36820 & .749 & 263.7 & 37910 & .931 & 267.7 & 37355 & .691 & × 262.0 & 38170 & .825 \\
\hline 1,3-Diethylbenzene & 271.8 & 36790 & .790 & 264.5 & 37810 & .927 & 268.3 & 37270 & .693 & 262.3 & 38120 & .875 \\
\hline 1,4-Diethylbenzene & 273.3 & 36590 & 1. 700 & 267.2 & 37420 & 1. 470 & a 270.0 & 37035 & .800 & 264.8 & 37765 & 1.505 \\
\hline
\end{tabular}

benzenes (see fig. 2) are distinctly different from those for the butylbenzenes, and they are more intense. The spectrum for 1,4-diethylbenzene differs noticeably from the other two diethylbenzenes. In general the two-spectra characteristic is less distinct, probably because of greater electronic migration, dissociation, and band splitting. The $A$ series is relatively weak, and in the 1,4-diethylbenzene the first band of the $A$ series is barely perceptible.

For comparative purposes the absorption spectrum of $n$-butylbenzene is displayed also with the diethylbenzenes. Here, again, it is interesting to note the gradual shift in the position of the important absorption bands in progressing from $n$-butylbenzene to 1,4-diethylbenzene. In this case the shift is toward the lower frequencies (longer wavelengths), resulting in the greatest shift for 1,4-diethylbenzene, although its spectrum is in some respects most like that for benzene of any of the substituents. A comparison of the two illustrations is interesting in displaying the gradual wavelength shift of the absorption bands in progressing through the seven substituted benzenes from 1,4-diethylbenzene to tert-butylbenzene.

The ratio of the specific absorbancies of the diethylbenzenes is in general agreement with the predictions of Sklar [11]. The value of absorbancy of the 1,4- spectrum is near twice that for the 1,2- and 1,3-substituents, as predicted. The spectra for both the butylbenzenes and the diethylbenzenes are in general agreement with other measurements upon similar materials both qualitatively and quantitatively where such data exist $[13,14,15]$.
In view of the similarity of the absorption spectra of these compounds, both in general appearance and in intensity, chemical analysis by ultraviolet absorption data alone would be impractical. The data should, however, serve a useful purpose in supplement to other measurements.

Acknowledgment is gratefully given to Nicolo Acquista for valuable assistance in the careful measurement of the concentrations of the various materials.

\section{References}

[1] Earle K. Plyler, Ralph Stair, and Curtis J. Humphreys, J. Research NBS 38, 211 (1947) RP1769.

[2] H. H. Cary and Arnold O. Beckman, J. Opt. Soc. Am. 31, 682 (1941).

[3] Kasson S. Gibson and Margaret M. Balcom, J. Research NBS 38, 601 (1947) RP1798.

[4] Supplement to NBS Circular C398, p. 7 (1949).

[5] Robert S. Mulliken, Chem. Rev. 41, 201 (1947).

[6] H. Sponer and E. Teller, Rev. Mod. Phys. 13, 76 (1941).

[7] G. Herzberg and E. Teller, Z. Physik Chem. B21, 410 (1933).

[8] H. Sponer, Chem. Rev. 41, 281 (1947).

[9] American Petroleum Institute Research Project 44. National Bureau of Standards. Catalog of ultraviolet spectrograms. Serial No. 1, Benzene, contributed by the Beacon Laboratory of the Texas Co.

[10] H. Sponer, G. Nordheim, A. L. Sklar, and E. Teller, J. Chem. Phys. 7, 207 (1939).

[11] A. L. Sklar, J. Chem. Phys. 7, 984 (1939); 10, 135 (1942).

[12] H. Sponer, and S. H. Wollman, J. Chem. Phys. 9, 816 (1941).

[13] F. A. Matsen, W. W. Robertson, and R. L. Chuoke, Chem. Rev. 41, 273 (1947). 
[14] American Petroleum Institute Research Project 44. National Bureau of Standards. Catalog of ultraviolet spectrograms. Serial No. $3, n$-Butylbenzene; Serial No. 4, Isobutylbenzene; Serial No. 5, secButylbenzene; Serial No. 6, tert-Butylbenzene, all contributed by the Ethyl Corp., Research Laboratories, Detroit, Mich. Serial No. 32, tert-Butylbenzene, Union Oil Co. of California. Serial No. 60, n-Butylbenzene; Serial No. 61, sec-Butylbenzene; Serial No. 69, Isobutylbenzene; Serial No. 70, tert-Butylbenzene, all contributed by the Beacon Laboratory of The Texas Co. Serial No. 165, Isobutylbenzene; Serial No. 166, both contributed by the Shell Development Co.

[15] American Petroleum Institute Research Project 44. National Bureau of Standards. Catalog of ultraviolet spectrograms. Serial No. 77, Ortho-diethyl- benzene; Serial No. 78, Meta-diethylbenzene; Serial No. 79, Para-diethylbenzene, all contributed by the Anglo-Iranian Oil Co., Ltd., Research Laboratories, Sunbury-on-Thames, Eng.

[16] American Petroleum Institute Research Project 44. National Bureau of Standards. Catalog of ultraviolet spectrograms. Serial No. 176, n-Butylbenzene; Serial No. 177, Isobutylbenzene; Serial No. 178, sec-Butylbenzene; Serial No. 179, tert-Butylbenzene; Serial No. 186, 1,2-Diethylbenzene; Serial No. 187, 1,3-Diethylbenzene; Serial No. 1,4-Diethylbenzene, all contributed by the Radiometry Laboratory, National Bureau of Standards, Washington, D. C.

Washington, August 6, 1948. 